CARTA AL EDITOR

\section{EVALUACIÓN DE LA EFICIENCIA DE FILTRACIÓN DE EQUIPOS DE PROTECCIÓN RESPIRATORIA CONTRA EL SARS-COV-2}

\section{EVALUATION OF THE FILTRATION EFFICIENCY OF RESPIRATORY PROTECTIVE EQUIPMENT AGAINST SARS-COV-2}

\author{
Manuel Chávez-Ruiz@1,a, Lenin Rueda-Torres@10,b, \\ Cristofer Bellido-Achahui(11,c, Frescia Madueño-Ventura@11,d, \\ Betsabé Ruffner-Camargo ${ }^{1, e}$ \\ 1 Centro Nacional de Salud Ocupacional y Protección del Ambiente para la \\ Salud, Instituto Nacional de Salud, Lima, Perú \\ a Licenciado químico; ${ }^{\mathrm{b}}$ licenciado tecnólogo médico; ${ }^{\mathrm{c}}$ licenciado químico \\ farmacéutico; ${ }^{\mathrm{d}}$ licenciada toxicóloga; ${ }^{\mathrm{e}}$ bachiller química
}

Sr. Editor: La evidencia muestra que una ruta importante de la transmisión del SARS-CoV-2 es a través de aerosoles y microgotas respiratorias $^{(1)}$. En ese sentido, las medidas preventivas para mitigar la cadena de contagios se han enfocado en cuarentenas, distanciamiento físico e higiene, complementado con el uso de equipos de protección respiratoria (EPR).

El uso de las mascarillas comunitarias supone limitar la transmisibilidad del virus. Si bien la evidencia de su efectividad en la población aún no es concluyente ${ }^{(2)}$, la capacidad de protección individual se debe a dos características básicas: la composición estructural (filtro) del material y el buen ajuste a la anatomía facial ${ }^{(3)}$. En la primera se evalúa la eficiencia de la filtración de partículas. Básicamente, el material se somete a pruebas con flujos de aerosol estándares asociados a partículas micrométricas, las cuales se cuantifican para establecer el porcentaje de retención del material ${ }^{(4)}$.

El Instituto Nacional para la Seguridad y Salud Ocupacional de los Estados Unidos (NIOSH, por sus siglas en inglés) establece la metodología de referencia (42 CFR 84) para la certificación de los respiradores N95. Dicho nombre corresponde, en efecto, a la capacidad de filtración de, al menos, el 95,0\% de partículas de $\mathrm{NaCl}$ de $0,3 \mu \mathrm{m}$ de diámetro. Asimismo, se manejan las categorías $\mathrm{R}$ (Resistant to oil) y P (for oil Proof) con sendas eficiencias de 95,9\% y hasta $99,9 \%{ }^{(5)}$.

Citar como: Chávez-Ruiz M, Rueda-Torres L, Bellido-Achahui C, Madueño-Ventura F, Ruffner-Camargo B. Evaluación de la eficiencia de filtración de equipos de protección respiratoria contra el Sars-CoV-2. Rev Peru Med Exp Salud Publica. 2020;38(3):467-8. doi: https://doi org/10.17843/rpmesp.2021.383.7564.

Correspondencia: Lenin Vladimir Rueda Torres; correo electrónico: lrueda@ins. gob.pe

Recibido: 11/03/2021 Aprobado: 25/08/2021 En línea: 29/09/2021
En cuanto a las mascarillas para uso comunitario, estas incluyen una variedad de tipos desechables o reutilizables, de fabricación propia o comercial, con productos textiles $\mathrm{u}$ otros materiales que permitan cubrir la boca, nariz y mentón. Debido a esta heterogeneidad, pocas combinaciones se han evaluado sistemáticamente $y$, en consecuencia, no tienen certificación. Al respecto, la Organización Mundial de la Salud recomienda que las mascarillas cuenten con tres capas en su confección (hidrófila, intermedia e hidrófoba). En el Perú, contamos con una resolución ministerial que detalla especificaciones oficiales para la confección de mascarillas para uso comunitario $^{(6)}$.

Frente a la gran demanda de mascarillas, diversas iniciativas promueven el desarrollo de alternativas de bajo costo que permitan evaluar el desempeño de los EPR no certificados ${ }^{(7)}$. Ello ha motivado un esfuerzo del Instituto Nacional de Salud, con el apoyo del Fondo Nacional de Desarrollo Científico Tecnológico y de Innovación Tecnológica (FONDECYT), para desarrollar un sistema de evaluación de la eficiencia filtrante de los EPR, a fin de obtener información y calcular objetivamente su capacidad de filtración, entre otros parámetros de desempeño, y poder así recomendar su uso y tomar decisiones basadas en evidencia.

El dispositivo desarrollado se fundamenta en la estructura básica de los equipos comerciales (Particle Filtration Efficiency Tester) reinventado con componentes análogos de bajo costo. Brevemente, se articularon un generador de aerosoles para aplicar el fluido $(\mathrm{NaCl}$ al $2 \%)$, una cámara de homogenizado, un sensor de presión diferencial, un sensor láser de alta precisión para el recuento de partículas, un caudalímetro de precisión y una serie de medidores de humedad y temperatura acoplados para el control de condiciones ambientales de trabajo; todos ellos controlados por un ordenador. Una vez colocada la muestra a evaluar (mascarilla) frente al flujo de ingreso, los sensores de medición extraen la información del recuento de partículas que atraviesan la membrana, la cual es tratada para determinar la eficiencia de filtración de partículas (EFP) mediante la siguiente fórmula: $\mathrm{EFP}=1$ - (número de partículas después de la muestra / número de partículas antes de la muestra) por 100.

El sistema permite calcular el porcentaje de filtración a diferentes tamaños de partículas $(0,3 ; 0,5 ; 1,0 ; 2,5 ; 5,0$ y $10 \mu \mathrm{m}$ ). Los parámetros técnicos de desempeño (anexo 1) se ajustan a especificaciones comparables con modelos comerciales, y el equipo permite estimar el índice de respirabilidad, un parámetro que valora la facilidad del paso del aire a través de la mascarilla.

La evaluación de tres elementos de protección respiratoria que se adquirieron en el mercado nacional muestra diferencias importantes de las eficiencias de filtración que proveen sus materiales. Como se observa en la Tabla 1, una mascarilla quirúrgica confiere más del triple de capacidad de retención de partículas en promedio respecto a una masca- 
Tabla 1. Evaluación de la eficiencia de filtración de partículas en tres elementos de protección respiratoria con aerosoles de $0,3 \mu \mathrm{m}$ de diámetro

\begin{tabular}{|c|c|c|c|c|}
\hline Muestra & Réplicas & $\begin{array}{c}\text { Promedio de partículas en } \\
\text { entrada (DE) }\end{array}$ & $\begin{array}{l}\text { Promedio de partículas filtradas } \\
\text { (DE) }\end{array}$ & $\begin{array}{r}\text { Eficiencia de } \\
\text { filtración (\%) }\end{array}$ \\
\hline Mascarilla quirúrgica $(\mathrm{n}=1)$ & 6 & $\begin{array}{c}458844,14 \\
(145883,31)\end{array}$ & $\begin{array}{l}15886,71 \\
(2122,95)\end{array}$ & 96,5 \\
\hline Mascarilla comunitaria $(\mathrm{n}=1)$ & 6 & $\begin{array}{c}959737,39 \\
(166075,61)\end{array}$ & $\begin{array}{c}661703,01 \\
(111941,41)\end{array}$ & 31,1 \\
\hline Tela de algodón $(\mathrm{n}=1)$ & 6 & $\begin{array}{c}1366284,54 \\
(82389,32)\end{array}$ & $\begin{array}{l}1101634,91 \\
(104673,98)\end{array}$ & 19,4 \\
\hline
\end{tabular}

DE: desviación estándar

rilla comunitaria simple de una sola capa, lo cual constituye un punto de partida para establecer especificaciones y valoración de riesgo.

Se concluye que el sistema desarrollado cumple el objetivo de estimar la eficiencia de filtración de EPR con diversos tamaños de partículas menores a $10 \mu \mathrm{m}$, las cuales se relacionan con tamaños de aerosoles respiratorios. Este dispositivo seguirá recolectando información útil que permitirá enfoques accesibles para la evaluación de emergencia

\section{REFERENCIAS BIBLIOGRÁFICAS}

1. Jarvis MC. Aerosol Transmission of SARS-CoV-2: Physical Principles and Implications. Front Public Heal. 2020;23;8:590041. doi: 10.3389/ fpubh.2020.590041.

2. European Centre for Disease Prevention and Control. Using face masks in the community: first update Effectiveness in reducing transmission of COVID-19 Key messages [Internet]. 2021. Disponible en: https:// www.ecdc.europa.eu/sites/default/files/documents/covid-19-face-masks-community-first-update.pdf.

3. Hill WC, Hull MS, MacCuspie RI. Testing of Commercial Masks and Respirators and Cotton Mask Insert Materials using SARS-CoV-2 Virion-Sized Particulates: Comparison of Ideal Aerosol Filtration Efficiency versus Fitted Filtration Efficiency. Nano Lett. 2020;20(10):7642-7.

4. ASTM F2299 / F2299M-03(2017). Standard Test Method for Determining the Initial Efficiency of Materials Used in Medical Face Masks to de mascarillas de uso comunitario de producción nacional y así contribuir con un sistema de evaluación que será de gran aporte para la lucha contra la pandemia en el Perú.

Financiamiento: Estudio financiado por FONDECYT.

Conflictos de interés: Los autores declaran no tener conflictos de interés.

Material suplementario: Disponible en la versión electrónica de la RPMESP.
Penetration by Particulates Using Latex Spheres, ASTM International [Internet]. West Conshohocken; 2017. Available from: www.astm.org.

5. National Institute for Occupational Safety and Health (NIOSH). NIOSH guide to the selection and use of particulate respirators certified under 42 CFR 84. [Internet]. 1996 Jan [cited 2021 Mar 4]. Disponible en: https://www.cdc.gov/niosh/docs/96-101/.

6. Ministerio de salud del Perú. RM No 135-2020-Minsa. Especificación Técnica para la confección de mascarillas faciales textiles de uso comunitario. 2020. p. 13.

7. Schilling K, Gentner DR, Wilen L, Medina A, Buehler C, Perez-Lorenzo LJ, et al. An accessible method for screening aerosol filtration identifies poor-performing commercial masks and respirators. J Expo Sci Environ Epidemiol [Internet]. 2020; Disponible en: doi: 10.1038/ s41370-020-0258-7. 